\title{
Conference Paper Study of Magnetic Property of Sn Doped Ni-Zn-Fe Nanoparticles
}

\author{
B. S. Tewari, Archana Dhyani, S. K. Joshi, Santosh Dubey, and Kailash Pandey \\ Department of Physics, University of Petroleum and Energy Studies, Dehradun 248007, India \\ Correspondence should be addressed to B. S. Tewari; bstewari@ddn.upes.ac.in
}

Received 5 February 2014; Accepted 11 March 2014; Published 9 April 2014

Academic Editors: R. Chandra, P. Mandal, R. K. Shivpuri, and G. N. Tiwari

This Conference Paper is based on a presentation given by B. S. Tewari at "National Conference on Advances in Materials Science for Energy Applications" held from 9 January 2014 to 10 January 2014 in Dehradun, India.

Copyright (C) 2014 B. S. Tewari et al. This is an open access article distributed under the Creative Commons Attribution License, which permits unrestricted use, distribution, and reproduction in any medium, provided the original work is properly cited.

The $\mathrm{Ni}_{0.6+x} \mathrm{Zn}_{0.4} \mathrm{Sn}_{x} \mathrm{Fe}_{2-2 x} \mathrm{O}_{4}(x=0.00$ to 0.04$)$ samples were prepared by solution route technique. These samples were characterized by XRD and EPR spectra at X-band frequency $(\sim 9.2 \mathrm{GHz})$. The XRD spectra of these ferrites confirm the formation of spinel structure. The average particle size calculated by using Scherrer's formula was found to be of the order of $24.7 \mathrm{~nm}$. The EPR spectra of these ferrites are mainly due to $\mathrm{Fe}^{3+}$ ions. $\mathrm{Fe}^{2+}$ ions have very short spin-lattice relaxation time and therefore EPR spectra of $\mathrm{Fe}^{2+}$ could be observed only at very low temperature. This fact is also supported by the isomer shift values of these ferrites obtained from Mössbauer spectroscopy. The variation of $g_{\mathrm{eff}}$ and $\Delta H_{\mathrm{PP}}$ with $\mathrm{Sn}^{4+}$ concentration is attributed to the variation of superexchange interaction. Moreover in this system the dominant process of relaxation is the spin lattice relaxation rather than the spin-spin interaction.

\section{Introduction}

Ferrites are certain double oxides of iron and another metal taken as the most important ferromagnetic substances. The magnetic ferrites fall mainly into two groups with different crystal structures. One is cubic ferrites having the general formula $\mathrm{MO} \cdot \mathrm{Fe}_{2} \mathrm{O}_{3}$ where $\mathrm{M}$ is a divalent metal ion, like $\mathrm{Mn}, \mathrm{Ni}, \mathrm{Fe}, \mathrm{Co}$, and $\mathrm{Mg}$. Second one is hexagonal ferrites. The most important member in this group is barium-ferrites $\mathrm{BaO} \cdot 6 \mathrm{Fe}_{2} \mathrm{O}_{3}$. The ferrites are ionic compounds, and their magnetic properties are due to the magnetic ions they contain. The commercial value lies in the fact that they have higher values of saturation magnetization and Curie temperature, which are imperative for use as core materials. They are more suitable for high power application in addition to the applications, such as multilayer chip inductor and electromagnetic interference (EMI) suppression; recent studies suggest their applications in biomedical applications such as molecular imaging and drug delivery [1-4].

There are two main classes of materials containing zinc ferrites, that is, Mn- $\mathrm{Zn}$ ferrites and Ni-Zn ferrites. Out of them Ni-Zn ferrites are designed for very high frequency operation, to more than $100 \mathrm{MHz}$ as well as very high resistivity, about $10^{5} \mathrm{ohm} \mathrm{cm}$. The effect of $\mathrm{TiO}_{2}$ addition on saturation magnetization and magnetic spectrum of $\mathrm{Ni}_{0.3} \mathrm{Zn}_{0.7} \mathrm{Fe}_{2} \mathrm{O}_{4}$ has been studied [4]. An unexpected dip was reported in the saturation magnetization curve at a particular $\mathrm{Ti}^{4+}$ concentration in $\mathrm{Ti}^{4+}$-substituted $\mathrm{Ni}-\mathrm{Zn}$ ferrite [5]. It has also been concluded that lattice parameter decreased with the increase of $\mathrm{Ti}^{4+}$ up to a certain concentration and subsequently it increased monotonically.

The variation of lattice parameter with high valent substitution is a combined effect of cation size on [6]:

(i) " $b$ "-the repulsion parameter for A site and

(ii) " $M$ "-Madelung Constant. He also observed a similar type of anomalous behavior in saturation curve for a wide range of Ti-substituted $\mathrm{Ni}-\mathrm{Zn}$ ferrites.

The present work is done to record and analyse the electronic paramagnetic resonance (EPR) spectra of Sn-substituted Ni$\mathrm{Zn}$ ferrite for various $\mathrm{Sn}$ concentrations at room temperature and liquid nitrogen temperature. The characterization of the prepared samples was done by XRD analysis. This work is a step towards studying the physical processes leading to anomalous behavior of magnetization in the Sn-substituted $\mathrm{Ni}-\mathrm{Zn}$ ferrites $[7,8]$. 


\section{Experimental Technique}

In the present work the EPR spectroscopy technique is employed. EPR spectroscopy studies the interaction of electron magnetic moment with a magnetic field and thus is applicable only to the systems having unpaired electrons (paramagnetic substances) or net angular momentum. EPR spectrum can be used to identify an unknown transition metal ion or lattice defect; it may be used to distinguish between the several valence states of the same ion. EPR spectrum frequently identifies the lattice site and symmetry of the paramagnetic species, particularly if single crystal data are available. Considerable information can be obtained about the nuclei in the immediate neighborhood of the absorbing spin and sometimes relaxation time data detects long range effects. The concentration of paramagnetic species may also be determined.

The important parameters from the EPR signal to be calculated are

(i) $g$-factor . It is calculated as

$$
g=2.00367 \frac{H_{\mathrm{DPPH}}}{H_{r}},
$$

where $H_{r}$ in the original absorption curve is the field corresponding to absorption (i.e., resonance field) for the given sample. In derivative curve it is measured.

(ii) Line width. It is defined as full width at half maximum (FWHM) for original absorption curve. For first derivative curve it is given by the peak-to-peak distance along magnetic field axis; that is,

$$
\Delta H_{\mathrm{pp}}=[H(\text { peak 2) }-H(\text { peak } 1)] .
$$

(iii) Spin-spin relaxation. It is calculated using the formula $1 / T_{2}=\pi g \mu_{B} \Delta H / h$, where $\Delta H$ is the FWHM (full width at half maximum) of the absorption peak or peak-to-peak line width of the first derivative curve.

\section{Results}

The EPR spectra of all the samples have an intense broad asymmetric peak having peak-to-peak line width $\Delta H_{\mathrm{pp}} \sim 2$ KGauss at room temperature. The spectra also have a very weak shoulder near zero field. The appearance of this weak shoulder near zero may be due to anisotropy effect. Here we are not interested in this particular investigation. Our discussion will be confined only for the peak corresponding to $g_{\mathrm{eff}} \sim 4$ for ferrites.

The graph plotted between $g_{\text {eff }}$ and the Sn concentration $x$ at room temperature is shown in Figure 1 . The $g_{\text {eff }}$ for base ferrite (i.e., $x=0.00$ ) is found to be 4.49 and it decreases to 2.98 up to $x=0.008$. It again increases to 3.21 at $x=0.012$ and then decreases to 2.95 up to $x=0.04$.

The variation of peak-to-peak line width $\Delta H_{\mathrm{pp}}$ as a function of " $x$ " in the $\mathrm{Ni}_{0.6+x} \mathrm{Zn}_{0.4} \mathrm{Sn}_{x} \mathrm{Fe}_{2-2 x} \mathrm{O}_{4}(x=0.00$ to 0.04 ) ferrite system at room temperature is shown in Figure 2. The value of $\Delta H_{\mathrm{pp}}$ for $x=0.00$ comes out to be 1.92 KGauss.

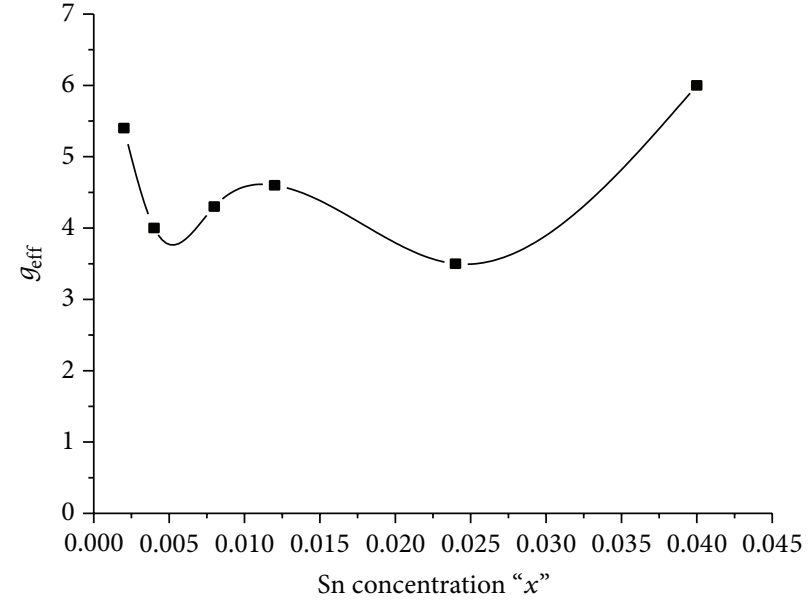

FIGURE $1: g_{\text {eff }}$ versus $\mathrm{Sn}$ concentration " $x$ " in $\mathrm{Ni}_{0.6+x} \mathrm{Zn}_{0.4} \mathrm{Sn}_{x} \mathrm{Fe}_{2-2 x} \mathrm{O}_{4}$ at RT.

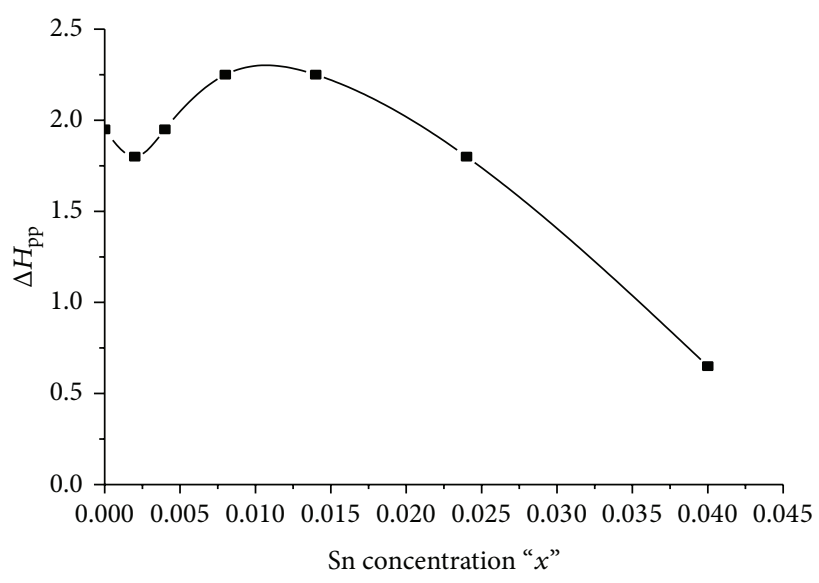

FIGURE 2: $\Delta H_{\mathrm{pp}}$ versus $\mathrm{Sn}$ concentration " $x$ " in $\mathrm{Ni}_{0.6+x} \mathrm{Zn}_{0.4} \mathrm{Sn}_{x} \mathrm{Fe}_{2-2 x} \mathrm{O}_{4}$ at RT.

It attains a maximum value of 2.225 KGauss at $x=0.008$ and at $x=0.012$. It then decreases monotonically to a value of 0.72 KGauss up to $x=0.04$. The variation of relaxation time $T_{2}$ with $\mathrm{Sn}$ concentration $x$ at room temperature is plotted in Figure 3. For the base ferrite, $\mathrm{Ni}_{0.6} \mathrm{Zn}_{0.4} \mathrm{Fe}_{2} \mathrm{O}_{4}$, the value of relaxation time comes out to be of the order of $2.63 \times 10^{-11}$ seconds at room temperature. Here we do not observe any systematic behaviour of relaxation time with $\mathrm{Sn}$ concentration. Previous workers [9-11] have also reported such smaller values of relaxation time for $\mathrm{Fe}^{3+}$ ions.

The magnetic interaction causes broadening of the resonance lines as the temperature is increased, if the interacting spins are alike, whereas it causes narrowing of resonance lines when unlike spins are involved. Since in $\mathrm{Ni}_{0.6+x} \mathrm{Zn}_{0.4} \mathrm{Sn}_{x} \mathrm{Fe}_{2-2 x} \mathrm{O}_{4}$ ferrite two different kinds of paramagnetic spins, namely, $\mathrm{Ni}^{2+}$ and $\mathrm{Fe}^{3+}$, are involved, the exchange interaction might be responsible for narrowing of the resonance peaks with increase of temperature.

The observed EPR spectra of the ferrite studied here is due to $\mathrm{Fe}^{3+}$ ions and not due to $\mathrm{Fe}^{2+}$ ions. The reason is that $\mathrm{Fe}^{2+}$ 


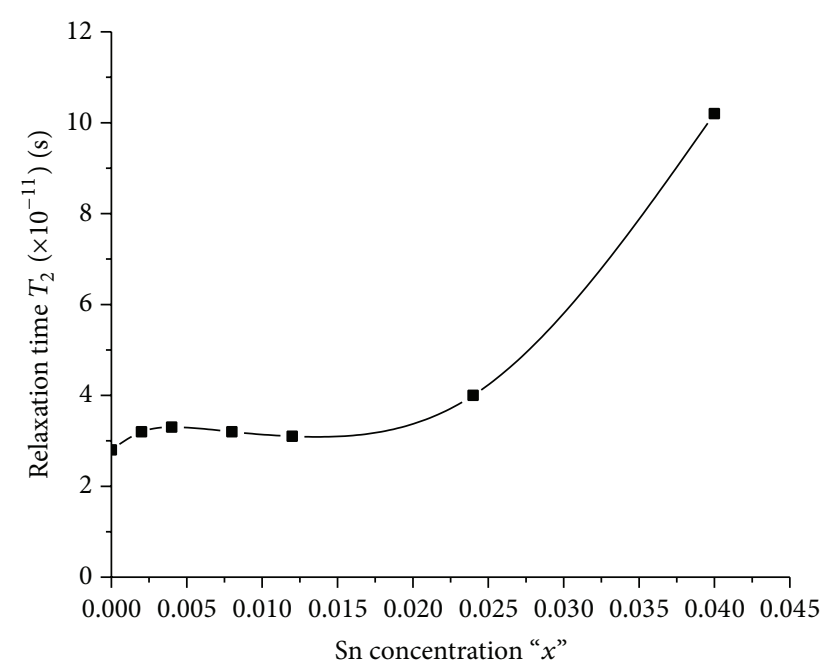

Figure 3: Relaxation time $T_{2}$ versus $\mathrm{Sn}$ concentration " $x$ ” in $\mathrm{Ni}_{0.6+x} \mathrm{Zn}_{0.4} \mathrm{Sn}_{x} \mathrm{Fe}_{2-2 x} \mathrm{O}_{4}$ at RT.

ions have very short spin lattice relaxation time and EPR can be observed at very low temperature (close to liquid Helium). Determination of isomer shift in this system of ferrite by ${ }^{57} \mathrm{Fe}$ Mössbauer spectroscopy confirmed the fact that iron is in $\mathrm{Fe}^{3+}$ state [12].

The value of $g_{\text {eff }}$ for bound unpaired electron is different than the value of $g_{\text {eff }}$ for a free electron. $g_{\text {eff }}$ for a bound electron depends on the magnetic interaction involving the orbital angular momentum of the unpaired electrons. Since the chemical environment of the unpaired electron changes the orbital angular momentum of the unpaired electron, therefore, one can say that $g_{\text {eff }}$ value likewise depends upon the chemical environment of the paramagnetic ions under consideration. The orbital angular momentum involves two types of magnetic interactions. One is the interaction of the orbital angular momentum with the electron spin, that is, the spin orbit coupling. The other interaction is that of the orbital angular momentum with the external magnetic field. The strength of these interactions affects the position and the width of the absorption peak in EPR spectra.

The occurrence of the finite line width in EPR spectrum is due to the fact that the electrons interact with externally applied magnetic field but they also interact magnetically with the surrounding of the samples. Thus the resultant magnetic field seen by a population of electron spins is not quite the same throughout the population even when they are subjected to the same applied field. Consequently resonance absorption line obtained for a given value of the resultant field will be obtained over a range of values of the applied field.

In many magnetic materials the EPR study has revealed that the variation of the resonance line width $\Delta H_{\mathrm{pp}}$ is caused by the microscopic magnetic interactions inside the material, mainly the interparticle magnetic dipole interaction and the superexchange interaction. In the case of ferromagnetic particles, the intrinsic molecular magnetic moments are large; therefore magnetic dipole interaction among these particles is very strong. Magnitude of this interaction is inversely proportional to the cube of average interparticle distance [13-15]. On other hand, superexchange interaction between magnetic ions through oxygen anions can reduce the value of $\Delta H_{\mathrm{pp}}$. The temperature dependence of relaxation time shows that, if line width is determined by spin lattice relaxation, it will decrease rapidly as temperature decreases. Further the elastic properties of these nanoparticles are currently being studied for their specific applications of high frequency devices [16].

\section{Conflict of Interests}

The authors declare that there is no conflict of interests regarding the publication of this paper.

\section{References}

[1] F. G. Brockman, V. D. Heide, and M. W. Louwerce, "Ferroxcube for proton synchrotrons," Phillips Technical Review, vol. 30, pp. 312-329, 1969.

[2] T. Nomura, "New evolution of ferrite for multilayer chip components," in Proceedings of 6th International Conference on Ferrites, vol. 6, p. 1198, Tokyo, Japan, 1992.

[3] T. Nakamura, "Snoek's limit in high-frequency permeability of polycrystalline Ni-Zn, Mg-Zn, and Ni-Zn-Cu spinel ferrites," Journal of Applied Physics, vol. 88, no. 1, pp. 348-353, 2000.

[4] Z. Liu, F. Kiessling, and J. Gatjens, "Another journal on nanomaterials?" Nanomaterials, vol. 1, pp. 1-2, 2010.

[5] D. C. Khan and M. Misra, "Magnetic, Mössbauer and electrical properties of Ti-substituted $\mathrm{Ni}_{0.3} \mathrm{Zn}_{0.7} \mathrm{Fe}_{2} \mathrm{O}_{4}$," Bulletin of Materials Science, vol. 7, no. 3-4, pp. 253-270, 1985.

[6] V. S. Ananthan, Effect of titatanium zirconium and tin on the variation of saturation magnetisation curie temperature and lattice parameter [M.S. thesis], Indian Institute of Technology, Kanpur, India, 1983.

[7] A. R. Das, V. S. Ananthan, and D. C. Khan, "Lattice parameter variation and magnetization studies on titanium-, zirconium, and tin-substituted nickel-zinc ferrites," Journal of Applied Physics, vol. 57, no. 8, pp. 4189-4191, 1985.

[8] R. C. Srivastava, D. C. Khan, and A. R. Das, "Mössbauer and magnetic studies of $\mathrm{Ti}^{4+}$-substituted Ni-Zn ferrites," Physical Review B, vol. 41, no. 18, pp. 12514-12521, 1990.

[9] R. C. Srivastava, D. C. Khan, A. R. Das, and T. M. Srinivasan, "Mössbauer and magnetic studies of titanium doped nickel ferrite," in Proceedings of the 5th International Conference on Ferrites, vol. 359, 1989.

[10] N. Bloembergen and S. Wang, "Relaxation effects in para- and ferromagnetic resonance," Physical Review, vol. 93, no. 1, pp. 7283, 1954.

[11] A. Upadhyay, Electron paramagnetic resonance study of $\mathrm{Ti}$ substituted $\mathrm{NiFe}_{2} \mathrm{O}_{4}$ [M.S. thesis], G. B. Pant University of Ag. \& Technology, Pantnagar, India, 2001.

[12] K. Pandey, Electron paramagnetic resonance study of Ti substituted Ni-Zn ferrite [M.S. thesis], G. B. Pant University of Ag. \& Technology, Pantnagar, India, 2003.

[13] D. C. Khan, R. C. Srivastava, and A. R. Das, "Mossbauer and magnetic studies of $\mathrm{Sn}^{4+}$-substituted Ni-Zn ferrites," Journal of Physics: Condensed Matter, vol. 4, no. 5, article 018, pp. 13791385, 1992. 
[14] T. Komatso, N. Soga, and N. Konagi, "ESR study of $\mathrm{NiFe}_{2} \mathrm{O}_{4}$ precipitation process from silicate glasses," Journal of Applied Physics, vol. 50, p. 6469, 1979.

[15] T. Komatso, N. Soga, and N. Konagi, "Superparamagnetic effects in the ferromagnetic resonance of silica supported nickel particles," The Journal of Chemical Physics, vol. 75, p. 5596, 1981.

[16] K. Praveena, K. Sadhana, and S. R. Murthy, "Elastic behaviour of Sn doped Ni-Zn ferrites," International Journal of Scientific and Research Publications, vol. 3, no. 2, 2013. 

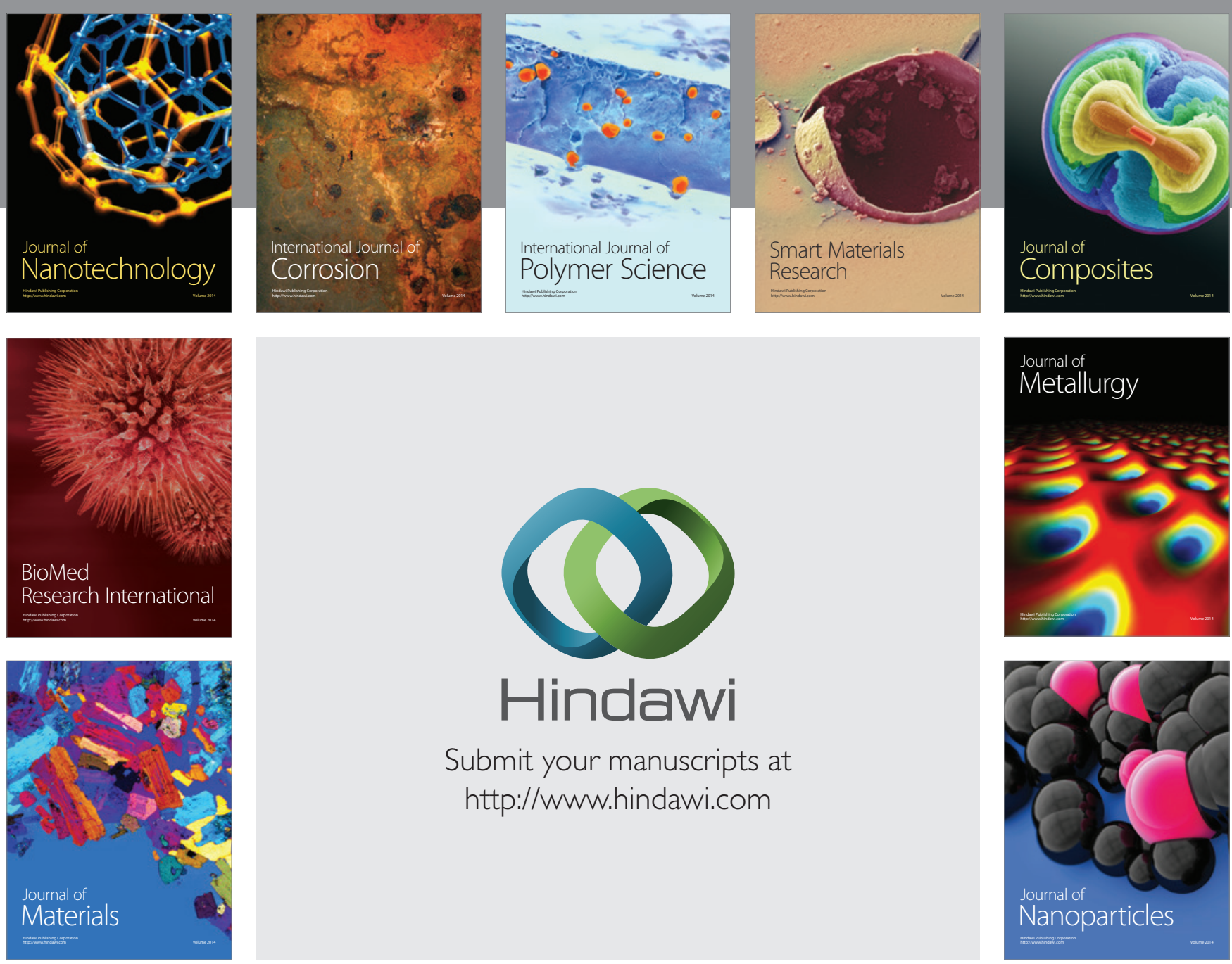

Submit your manuscripts at http://www.hindawi.com
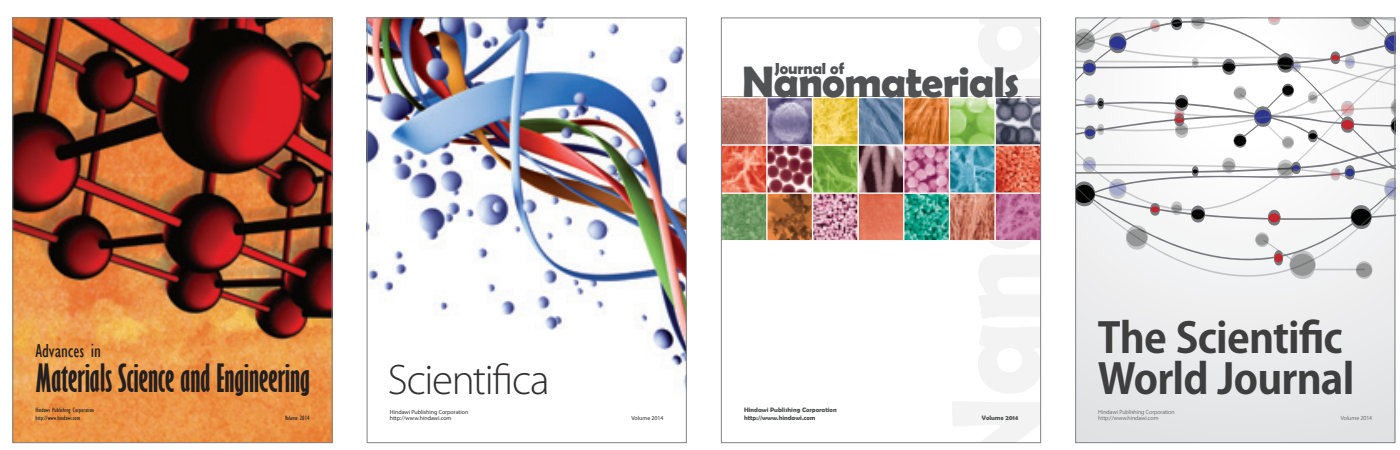

\section{The Scientific World Journal}
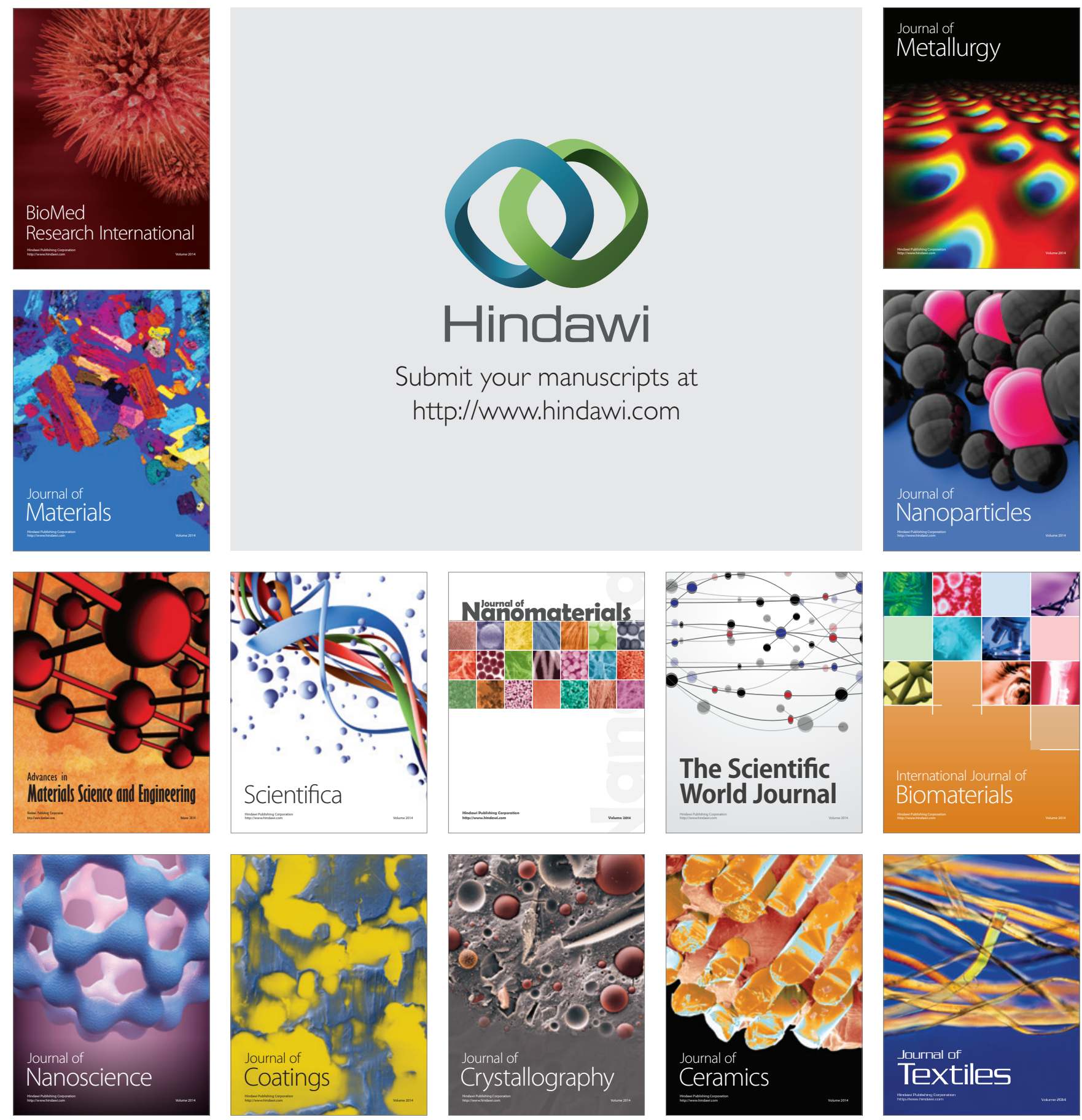